\title{
Research Square \\ Comparison between the Gravitational Redshift in the Kerr and Schwarzschild Fields
}

Charles McGruder ( $\square$ mcgruder@wku.edu )

Western Kentucky University

\section{Research Article}

Keywords: general relativity, neutron stars, white dwarfs

Posted Date: March 16th, 2021

DOI: https://doi.org/10.21203/rs.3.rs-276860/v1

License: (c) (i) This work is licensed under a Creative Commons Attribution 4.0 International License. Read Full License 


\title{
Comparison between the Gravitational Redshift in the Kerr and Schwarzschild Fields
}

\author{
Charles H. McGruder III \\ Department of Physics and Astronomy, Western Kentucky University, Bowling Green, KY 42101 \\ Received: $\quad$; Accepted:
}

\begin{abstract}
The Schwarzschild and Kerr metrics are solutions of Einstein field equations of general relativity representing the gravitational fields of a non-rotating spherical mass and a rotating black hole respectively. Unlike the Kerr field, the gravitational redshift in the Schwarzschild field is well known. We employ the concept of stationary clocks to derive the gravitational redshift in the Kerr field demonstrating that frame dragging plays no role. We then calculate the Kerr gravitational redshift for the earth, sun, white dwarfs and neutron stars and compare them with the Schwarzschild gravitational redshift, showing that the gravitational redshift on earth and from the sun does not differ from the Schwarzschild gravitational redshift. For extreme cases of rapidly rotating white dwarfs and neutron stars there is a significant difference between the two gravitational redshifts. Unlike the Schwarzschild gravitational redshift, the Kerr gravitational redshift has to date not been put on a firm observational basis. We point out that the gravitational redshift in the Kerr field possess a latitude dependency, which cannot be confirmed through solar or terrestrial observations, but can be on rapidly rotating white dwarfs and neutron stars.
\end{abstract}

Keywords: general relativity, neutron stars, white dwarfs 


\section{Introduction}

On theoretical grounds as far back as 1907 Einstein predicted that clocks run slower in a gravitational field. He concluded from this circumstance that light emitted from the sun will be shifted toward longer wavelengths. Today we call this phenomenon gravitational redshift. In 1911 Einstein returned to this topic. Employing the equivalence principle he derived the equation that governs gravitational redshift from the corresponding expression for the slowing down of time in a gravitational field. These publications appeared before Einstein completed his theory of general relativity in November 1915. Shortly thereafter, in January 1916, Schwarzschild published his solution to Einstein's field equations, which describes the gravitational field outside a spherical non rotating mass. In December 1916 Johannes Droste, not knowing of Schwarzschild's work, published the first Ph.D. thesis on general relativity (McGruder \& VanderMeer 2018), in which he presented a more elegant derivation of the Schwarzschild solution, which is closer to the modern approach. It is common in textbooks (for instance Weinberg 1972) to derive the gravitational redshift in the Schwarzschild field from time dilation of stationary clocks.

Observationally, gravitational redshift was first claimed to be detected by Adams in 1925 and confirmed by Moore (1928) on Sirius B, which is a white dwarf. However, (Hetherington 1980; Greenstein et al.1985; Wesemael 1985; Holberg 2010) showed that these early results were erroneous. Ground-based experiments by Pound \& Rebka 1960 and Pound \& Snider 1965 and a space-born experiment by Vessot et al. 1980 agreed with the predictions of the equivalence principle (Einstein 1911), while others (Blamont \& Roddier 1961; Snider 1970, 1972, 1974; Lapresto Chapman \& Sturgis 1979; Takeda \& Ueno 2012) measured the gravitational redshift in sunlight confirming the earlier predictions of Einstein. In addition astronomers have measured the gravitational redshift in white dwarfs (Wegner 1980; Wegner 
\& Reid 1991; Falcon et al. 2010; Pasquini et al. 2019; Greenstein \& Trimble 1967; Trimble \& Greenstein 1972; Koester 1987; Wegner 1989; Oswalt et al. 1991; Reid 1996; Koester \& Weidemann 1991; Barstow er al. 2005; Holberg et al. 2012; Parsons et al. 2012; Parsons et al. 2017; Greenstein, Oke \& Shipman 1971; 1985; Wesemael 1985), and in neutron stars (Hambaryan et al. 2017 and Tang et al. 2020)

The Schwarzschild metric is applicable to the earth, sun, and white dwarfs because they do not rotate rapidly, so the effect of rotation on their gravitational redshifts is not significant as we show below. Neutron stars however possess very high rates of rotation. Nevertheless, recent work on neutron stars (Bogdanov et al. 2019) employs the Schwarzschild and not the Kerr gravitational redshift. Is it justified to employ the Schwarzschild gravitational redshift in neutron star work?

The purpose of our work is to derive the gravitational redshift in the Kerr field. We consider the simplist case of a emitter and receiver, which are both static. They therefore follow the integral curves of the timelike Killing vector field in both the Schwarzschild and Kerr spacetimes. We demonstrate that frame dragging plays no role in the derivation. We apply our expression to ground-based experiments, the sun, white dwarfs and neutron stars. We show that it is indeed justified not to employ the Kerr gravitational redshift in most neutron star work because the Kerr and Schwarzschild gravitational redshifts are equal. However, in cases of neutron stars with very high angular velocities, the gravitational redshifts are not equal. In such cases presumably the Kerr metric or generalizations (Hartle \& Thorne 1968; Quevedo \& Mahhoon 1991) provide a more accurate description of the gravitational redshift. 


\section{Theory}

In this section we obtain expressions for the gravitational redshift in both the Schwarzschild and Kerr fields. We start with the covariant form of the metric tensor with signature (+,-,-,-):

$$
d s^{2}=g_{t t} c^{2} d t^{2}+g_{r r} d r^{2}+g_{\theta \theta} d \theta^{2}+g_{\phi \phi} d \phi^{2}+2 g_{t \phi} c d t d \phi
$$

where $r, \theta$ and $\phi$ are spherical coordinates. $d s$ is the line element, $t$ time coordinate, $r$ radial coordinate, $\theta$ colatitude or polar coordinate $(0 \leq \theta \leq \pi)$, and $\phi$ the azimuthal coordinate or longitude ( $0 \leq \phi \leq 2 \pi$ ). The speed of light is $c$. Equation 1 is valid for both the Schwarzschild and Kerr metrics. They differ in the specific expressions for the components of the metric tensor, $g_{\mu \nu}$.

Following president we first derive the gravitational time dilation to obtain the gravitational redshift. It is given for a clock that is stationary (motionless) in a gravitational field. Stationary means:

$$
d r=d \theta=d \phi=0
$$

Equation 1 reduces to:

$$
d s^{2}=g_{t t} c^{2} d t^{2}
$$

where $d s$ is called the proper time and $d t$ the coordinate time. Equation 3 leads to the time, $d \tau$, of a stationary clock in the gravitational field (Fließbach 2006):

$$
d \tau=\frac{d s}{c}=\sqrt{g_{t t}} d t
$$

Equation 4 is valid for all metrics ${ }^{1}$ including the Schwarzschild and Kerr metrics.

It is not possible to determine $d \tau$ at any specific point in the gravitational field because all devices are affected in exactly the same way. Thus, two points in the gravitational field

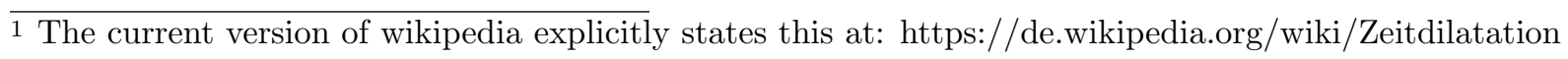


are required to determine the influence of $g_{t t}$. As clocks we consider atoms, which emit monochromatic electromagnetic radiation at specific frequencies. Consider two points in the gravitational field, point A, where electromagnetic radiation of a specific frequency is emitted and point $B$, where it is received. We have from equation 4:

$$
\begin{aligned}
& d \tau_{A}=\sqrt{g_{t t}\left(r_{A}\right)} d t_{A} \\
& d \tau_{B}=\sqrt{g_{t t}\left(r_{B}\right)} d t_{B}
\end{aligned}
$$

As the time intervals, $d \tau_{A}$ and $d \tau_{B}$, we assign the time between adjacent crests of electromagnetic radiation. This means they are equal to the reciporal of the period or frequency, $\nu_{A}$ and $\nu_{B}$, of the electromagnetic radiation. That is:

$$
d \tau_{A}=\frac{1}{\nu_{A}}
$$

and

$$
d \tau_{B}=\frac{1}{\nu_{B}}
$$

From equations 5, 6, 7 and 8, it follows that:

$$
\frac{\nu_{A}}{\nu_{B}}=\sqrt{\frac{g_{t t}\left(r_{B}\right)}{g_{t t}\left(r_{A}\right)}}
$$

The definition of the redshift, $z$, is:

$$
z=\frac{\nu_{A}}{\nu_{B}}-1=\frac{\lambda_{B}}{\lambda_{A}}-1
$$

where $\lambda$ is the wavelength of the electromagnetic radiation. Equations 9 and 10 lead to the gravitational redshift, $z$ :

$$
z=\sqrt{\frac{g_{t t}\left(r_{B}\right)}{g_{t t}\left(r_{A}\right)}}-1
$$


We emphasize that Equation 11 is valid for all metrics including the Schwarzschild and Kerr metrics. In the Schwarzschild field $g_{t t}$ is:

$$
g_{t t}=1-\frac{r_{s}}{r}
$$

where $r_{s}$ is the Schwarzschild radius. With $M$ the mass and $G$ the gravitational constant $r_{s}$ is:

$$
r_{s}=\frac{2 G M}{c^{2}}
$$

Inserting equation 12 into equation 11 leads to the gravitational redshift, $z_{s}$, in the Schwarzschild field:

$$
z_{s}=\sqrt{\frac{1-\frac{r_{s}}{r_{B}}}{1-\frac{r_{s}}{r_{A}}}}-1
$$

if $r_{B} \gg r_{s}$ and $r_{B} \gg r_{A}$, then the above equation reduces to:

$$
z_{s}=\sqrt{\frac{1}{1-\frac{r_{s}}{R}}}-1
$$

where we have replaced $r_{A}$ with $R$, the radius of Earth or the celestial body being considered.

In the Kerr field $g_{t t}$ is:

$$
g_{t t}=1-\frac{r_{s} r}{r^{2}+a^{2} \cos (\theta)}
$$

where $a$ is related to the angular momentum, $J$, and mass, $M$ via:

$$
a=\frac{1}{c} \frac{J}{M}
$$

We will employ the classical formula for the angular momentum of a rotating sphere to compute $J$ :

$$
J=\frac{2}{5} M R^{2} \Omega
$$

Where $R$ is the radius of the sphere (that is the Earth or celestial body being considered) and $\Omega$ its angular velocity. Inserting equation 18 into equation 17 leads to:

$$
a=\frac{2}{5} \frac{\Omega}{c} R^{2}
$$


whereby $\Omega$ is given via the rotational period, $P$, by:

$$
\Omega=\frac{2 \pi}{P}
$$

Inserting equation 16 into equation 11 yields the gravitational redshift, $z_{k}$, in the Kerr field:

$$
z_{k}=\sqrt{\frac{1-\frac{r_{s} r_{B}}{r_{B}^{2}+a^{2} \cos (\theta)}}{1-\frac{r_{s} r_{A}}{r_{A}^{2}+a^{2} \cos (\theta)}}}-1
$$

We note that if $a=0$, then the $g_{t t}$ in the Kerr field, Equation 16, reduces to the $g_{t t}$ in the Schwarzschild field, Equation 12, and $z_{k}$ becomes $z_{s}$. If $\theta=\frac{\pi}{2}$, that is the point of emission is on the equator, then $\cos (\theta)=0$, which also leads to $z_{k}=z_{s}$. If $r_{A}^{2} \gg a^{2} \cos (\theta)$ and $r_{B}^{2} \gg a^{2} \cos (\theta)$ then $z_{k}$ reduces to $z_{s}$. If $r_{B}^{2} \gg a^{2} \cos (\theta)$ and $r_{B} \gg r_{A}$ then $z_{k}$ becomes:

$$
z_{k}=\sqrt{\frac{1}{1-\frac{R r_{s}}{R^{2}+a^{2} \cos (\theta)}}}-1
$$

Our expressions for $z_{k}$ differ from that of Dubey and Sen (2015). They are dependent on the polar coordinate, $\theta$, only, whereas their expression depends on both $\theta$ and $\phi$. They obtained a different expression primarily because they assumed $d \phi \neq 0$, that is frame dragging is involved in the gravitational redshift. This assumption was presumably made because

real physical objects must experience frame dragging, whereas our expression is derived from time dilation associated with stationary, Equation 2, clocks. Their expression, however, leads them to conclude that the sun exhibits very high values of redshift, which are not observed. We elaborate on this circumstance in the next section.

\section{Comparison of Theory with Observations}

In this section we compute the Schwarzschild and Kerr gravitational redshifts for the earth, sun, white dwarfs and neutron stars and compare them with observations, if available. 


\subsection{Earth}

First we neglect the earth's rotation and employ the Schwarzschild gravitational redshift to predict the ground-based results of Pound and Snider (1965). The radius of the earth is: $r_{A}=6378.137 \mathrm{~km}$. The height of the tower in the Pound and Snider experiment is 75 feet, so $r_{B}=r_{A}+0.02286 \mathrm{~km}$. Equation 13 yields the Schwarzschild radius of the earth: $r_{s}=8.8701 \times 10^{-6} \mathrm{~km}$. Inserting these values into Equation 14 gives: $z_{s}=2.44249 \times 10^{-15}$. If we include the earth's rotation, $\Omega=7.292115 \times 10^{-5} \mathrm{~s}^{-1}$, by employing equation 19 we are lead to: $a=0.0395804 \mathrm{~km}$. Inserting $a$ into Equation 21 we obtain the exact same value namely:

$$
z_{s}=z_{k}(\theta=0)=z_{k}\left(\theta=\frac{\pi}{2}\right)=z_{k}(\theta=\pi)=2.44249 \times 10^{-15}
$$

This means that the rotation of the earth plays no role in the Pound and Snider groundbased experiment, which is the case because $a$ is so small. The corresponding value obtained by Pound and Snider is: $2.4525 \times 10^{-15}$. Thus, theory and experiment are in close agreement.

\subsection{Sun}

To compute the Schwarzschild gravitational redshift we note that the radius of the sun is: $r_{A}=6.957 \times 10^{5} \mathrm{~km}$. We take the astronomical unit to be the mean distance of the earth from the sun: $r_{B}=1.495978707 \times 10^{8} \mathrm{~km}$. Finally, with the mass of the sun being $M_{\odot}=1.98847 \times 10^{33} \mathrm{~g}$, equation 13 gives the Schwarzschild radius, $r_{s}=2.95334 \mathrm{~km}$. Inserting these values into Equation 14 yields: $z_{s}=2.11075 \times 10^{-6}$, which is nine orders of magnitude larger than that in the Pound and Snider ground-based experiment.

Next we compute the Kerr gravitational redshift for the sun. The rotation of the sun is complicated because the solar surface performs differential rotation and the core rotates faster than the surface. We assume that $\Omega=2.5982 \times 10^{-6} \mathrm{~s}^{-1}$ giving us via equation 
19: $a=1.68095 \mathrm{~km}$. Applying equation 21 we get the same value for both the Kerr and Schwarzschild gravitational redshifts:

$$
z_{s}=z_{k}(\theta=0)=z_{k}\left(\theta=\frac{\pi}{2}\right)=z_{k}(\theta=\pi)=2.11075 \times 10^{-6}
$$

Thus, like the earth, the rotation of the sun plays no role in the gravitational redshift. Again this is because the value of $a$ is small. Thus, we cannot confirm the validity of the Kerr gravitational redshift via solar or terrestrial observations.

Many authors listed in the introduction obtained results at or very close to the above redshift. The latest measurement(Takeda \& Ueno 2012) of the solar gravitational redshift is "consistent" with $633 \mathrm{~m} / \mathrm{s}$, which according to the well known non-relativisic Doppler formula is:

$$
z=\frac{\triangle v}{c}=\frac{633}{c}=2.11 \times 10^{-6}
$$

Takeda and Ueno obtained their result by using 3,000 data points over the entire solar disk. This result as well as those of other authors is in complete agreement with our Schwarzschild and Kerr gravitational redshifts and is in contrast to those of Dubey and Sen (2015), who find that $z_{k}>300$ on the equator at $\phi \approx 90^{\circ}$ and $\phi \approx 270^{\circ}$.

\subsection{White Dwarfs}

It is clear from Equation 22 that $a$ and therefore the Kerr metric is only going to play a significant role, when the angular velocity, $\Omega$ is high. White dwarfs are slowly rotating degenerate stars, with periods generally between $1 h$ and $18 d$ with a median of approximately $1 d$ (Kepler 2017). There are only a few white dwarfs known with significantly shorter

periods. For instance, we follow Malheiro et al. (2012) and consider the soft gamma ray repeator, SGR $0418+5729$, to be a white dwarf with $M=1.4 M_{\odot} R=10^{3} \mathrm{~km}$. These values lead to a Schwarzschild gravitational redshift: $z_{s}=2.07377 \times 10^{-3}$. SGR $0418+5729$ has a 
very short period (Malheiro et al. 2012) of: $P=9.08$ s. Plugging $P$ into Equation 20 and then employing Equation 19 yields: $a=0.92328 \mathrm{~km}$. Now we use Equation 22 to calculate the Kerr gravitational redshift, which turns out to have the same value as the Schwarzschild gravitational redshift:

$$
z_{s}=z_{k}(\theta=0)=z_{k}\left(\theta=\frac{\pi}{2}\right)=z_{k}(\theta=\pi)=2.07377 \times 10^{-3}
$$

Thus, even if we believe that SGR $0418+5729$ is a white dwarf with such an extremely short period for a white dwarf, the Kerr metric plays no role in the gravitational redshift.

General relativistic treatments of white dwarfs has been carried out by Rotondo et al. (2011) for non rotating and by Boshkayev et al. (2011) for rotating white dwarfs. Their work takes into account nuclear, weak and electromagnetic interactions and modifies the classical Chandrasekhar limit of white dwarfs masses, $M \approx 1.4 M_{\odot}$, which is based on special relativity. Boshkayev et al. (2011) obtain a maximum mass of white dwarfs: $M=1.51595$ $M_{\odot}$, with an equatorial radius of $R=1583.47 \mathrm{~km}$ and a minimum rotational period of: $P=0.3 \mathrm{~s}$. These values lead to a Schwarzschild gravitational redshift of: $z_{s}=1.41671 \times 10^{-3}$ and as depicted in Figure 1 to the Kerr values of: $z_{k}(\theta=0)=1.41393 \times 10^{-3}, z_{k}\left(\theta=\frac{\pi}{2}\right)=$ $1.41671 \times 10^{-3}$ and $z_{k}(\theta=\pi)=1.41949 \times 10^{-3}$, meaning that $\Delta\left(z_{k}(\theta=\pi)-z_{k}(\theta=0)\right)=$ $5.56 \times 10^{-6}$. Some of the latest work on gravitational redshifts of white dwarfs (Pasquini et al. 2019) achieves $0.01 \mathrm{~km} / \mathrm{s}$ precision, which according to Equation 23 corresponds to $\Delta z=3.3 \times 10^{-8}$. No such white dwarfs have been discovered yet, but if found they may allow us to differentiate between the Schwarzschild and Kerr gravitational redshifts.

\subsection{Neutron Stars}

The fiducial mass and radius of neutron stars are well-known to be: $M=1.4 M_{\odot}$ and $R=10 \mathrm{~km}$. We use this value of $M$ in Equation 13 to obtain the Schwarzschild radius 
of: $r_{s}=4.3468 \mathrm{~km}$. According to Equation 15 the Schwarzschild gravitational redshift is: $z_{s}=0.305732$

The fastest rotating neutron star known is PSR J1748-2446ad, which completes 716 rotations in a second and according to Equation 20 corresponds to: $\Omega=4.49875 \times 10^{3}$ $s^{-1}$. It follows from Equation 22 that $z_{k}(\theta=0)=0.304083, z_{k}\left(\theta=\frac{\pi}{2}\right)=0.305732$ and $z_{k}(\theta=\pi)=0.307399$ meaning that $z_{k}=z_{s}$ on the equator and $\Delta\left(z_{k}(\theta=\pi)-z_{k}(\theta=\right.$ $0))=0.003316$. Our results differ strikingly from those of Dubey and Sen (2015), who find a double peaked distribution for this source as a function of longitude, $\phi$. Their peaks amount to: $z \approx 1.6$ and $z \approx 1.2$. According to them our value of $z_{k} \approx 0.3$ is only found at four values of $\phi$.

Kaaret et al. (2007) however found strong evidence that the X-ray emitting neutron star: XTE J1739-285 has an even higher rotational frequency than PSR J1748-2446ad, namely $1125 \mathrm{~Hz}$. As figure 2 depicts, this frequency leads to: $z_{k}(\theta=0)=0.301714, z_{k}\left(\theta=\frac{\pi}{2}\right)=$ 0.305732 and $z_{k}(\theta=\pi)=0.309859$ meaning that $z_{k}=z_{s}$ on the equator and $\Delta\left(z_{k}(\theta=\right.$ $\left.\pi)-z_{k}(\theta=0)\right)=0.008145$. Hambaryan et al. (2017) measured the gravitational redshift of the isolated neutron star, RX J0720.4-3125, to be: $z=0.205_{-0.003}^{+0.006}$. This result suggests that it may be possible to differentiate between the Schwarzschild and Kerr gravitational redshifts of very rapidly rotating neutron stars, possibly allowing us to put the Kerr gravitational redshift on a firm observational basis.

\section{Conclusion}

We have derived an expression for the gravitational redshift in the Kerr field, which is the field corresponding to a rotating black hole. We applied our result to earth based experiments, the sun, white dwarfs and neutron stars. We compared our results with the 
Schwarzschild gravitational redshift. We found that the two redshifts do not differ for the earth, sun, white dwarfs and the vast majority of neutron stars. Only for the fastest rotating neutron stars is their a significant difference between the two redshifts and also for the fastest possible general relativistic white dwarfs. We conclude that the influence of rotation on the gravitational redshift associated with the earth, sun and all white dwarfs discovered to date and the vast majority of neutron stars is completely negligible. meaning that in these cases the simpler expression for the Schwarzschild gravitational redshift can be employed.

\section{REFERENCES}

Barstow, M.A., et al. 2005, MNRAS, 362, 1134

Blamont, J. E., Roddier, F., 2061, PhRvL, 7, 437

Bogdanov, S., et al., 2019, ApJL, 887, L26

Boshkayev, J. R., Rueda, J., Ruffini, R., 2011, 20, 136

Dubey, A. K., Sen, A. K., 2015, IJTP, 54, 2398

Einstein, A., 1907, JRE, 4, 411

Einstein, A., 1911, AnP, 340, 89

Fließbach, T., 2006, Allgemeine Relativitätstheorie, Spektrum akademischer Verlag, ISBN:-13: 978-3-8274-1685-8

Greenstein, J. L., Trimble, V. L., 1967, ApJ, 149, 283

Greenstein, J. L., Oke, J. B., Shipman, H. L., 1971, ApJ, 169, 56

Greenstein, J. L., Oke, J. B., Shipman, H. L., 1985, QJRAS, 26, 279

Hambaryn, V. Suleimanov, F., Haberl, F., et al., 2017, A\&A, 601, A108

Hartle, J.B. Thorne, K.S., 1968, ApJ, 153, 807

Hessels, J. W. T., Ransom, S. M., Stairs, I. H., 2006AAS 207, id 209.07

Hetherington N. S., 1980, QJRAS, 21, 246

Holberg, J. B.. 2012, AJ, 143, 68

Holberg, J. B., 2010, JHA, 41, 41

Kaaret, P., Prieskorn, Z., In't Zand, J. J. M., et al., 2007,ApJ, 657, L100

Koester, D., 1987, ApJ,322, 852

Koester, D., Weidemann, V., 1991, AJ, 102, 1152 
LoPresto, J. C., Schrader, C., Pierce A. K., 1991, ApJ, 376, 757

LoPresto, J. C., Chapman, R. D., Sturgis, E. A. 1980,SoPh, 66, 245

Malheiro, M., Rueda, J. A., Ruffini, R., 2012, PASJ, 64, id 56

McGruder, C. H. \& VanderMeer, B. W., 2018, arXiv:1801.075921

Oswalt, T. D., Sion, E. M., Hammond, G., et al., 1991,AJ, 101, 583

Parsons, S. G., Marsh, T. R., Gänsicke, B. T., 2012, MNRAS, 420, 3297

Parsons, S. G., Marsh, T. R., Gänsicke, B. T., 2017, MNRAS, 470, 4473

Pasquini, L. Pala, A. F., Ludwig, H. G., et al., 2019, A\&A, 627, L8

Pound, R. V. \& Rebka, G. A., 1960, PhRvL, 4, 337

Pound, R. V. \& Snider, J. L., 1965, PhRv, 140, B 788

Quevedo, H. Mashoon, B. 1991, PhRv D, 43, 3902

Reid, I. N., 1996, AJ, 111, 2000

Rotondo, M., Rueda, J. A., Ruffini, R., Xue, S. 2011, PhRvD, 84, id 084007

Schwarzschild, K., 1916, Sitzungsberichte der Königlich Preussischen Akademie der Wissenschaftern zu Berlin, 189

Snider, J. L., 1970, SoPh, 12, 352

Snider, J. L., 1971, SoPh, 12, 352

Snider, J. L., 1974, PhRvL, 28, 853

Takeda, Y., \& Ueno, S., 2012, SoPh, 281, 551

Tang, S., Jiang, J., Gao, W., et al., 2020, ApJ, 888, id 45

Trimble, V. \& Greenstein, J. 1972, ApJ, 177, 441

Vessot, R. F. C., Levine, M. W. Mattison, E. M., 1980, PhRvL, 45, 2081

Wegner, G., 1980, AJ, 85, 1255

Wegner, G., Reid, I. N., McMahan, R. K., 1991, ApJ, 376, 186

Weinberg, S., 1972, Gravitation and Cosmology, John Wiley and Sons, Inc., ISBN: 0-471-92567-5 
Fig, 2: z vs. $\theta$ for neutron star, XTE J1739-285

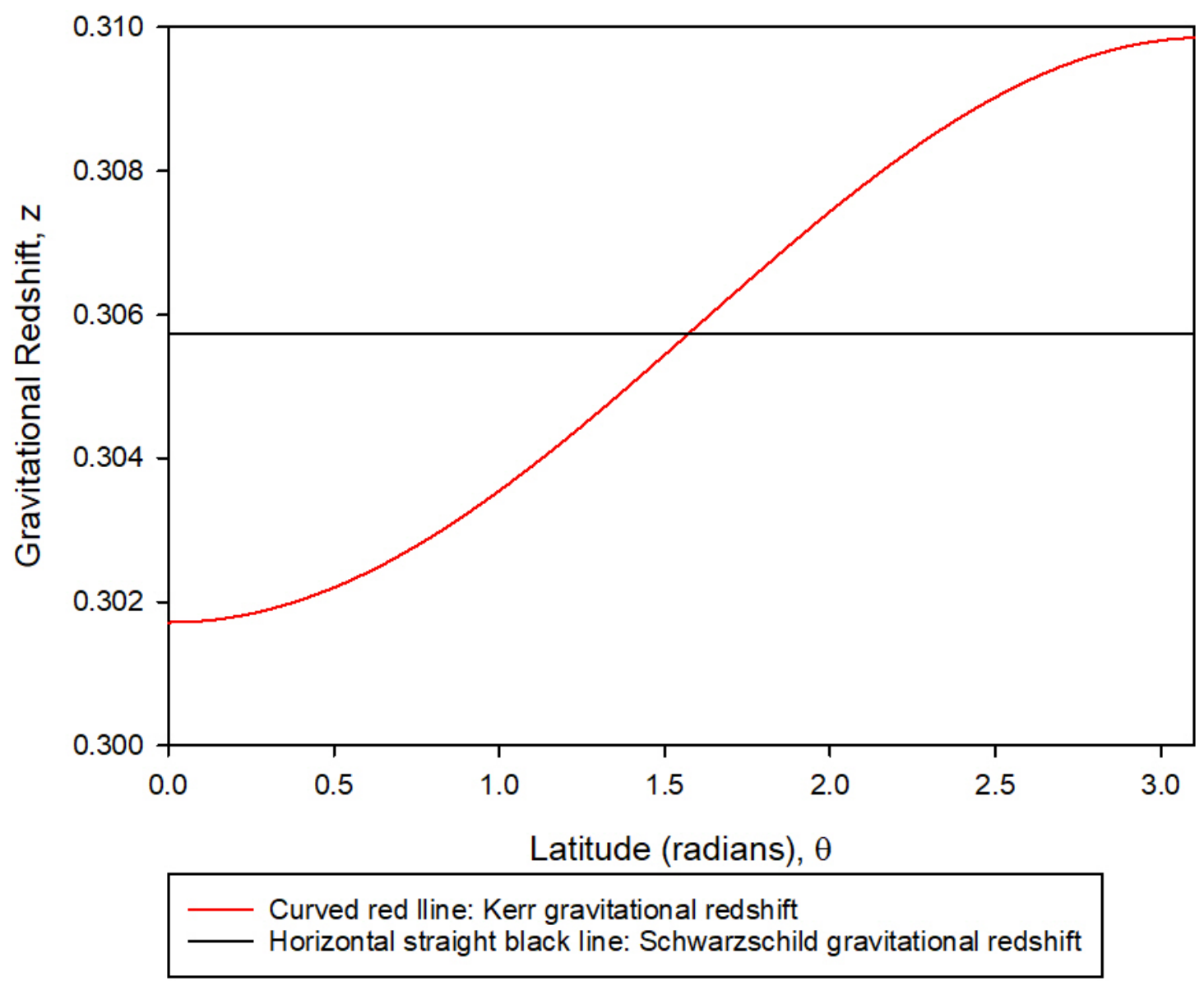


Figures

Fig. 1: z vs. $\theta$ for general relativistic white dwarf

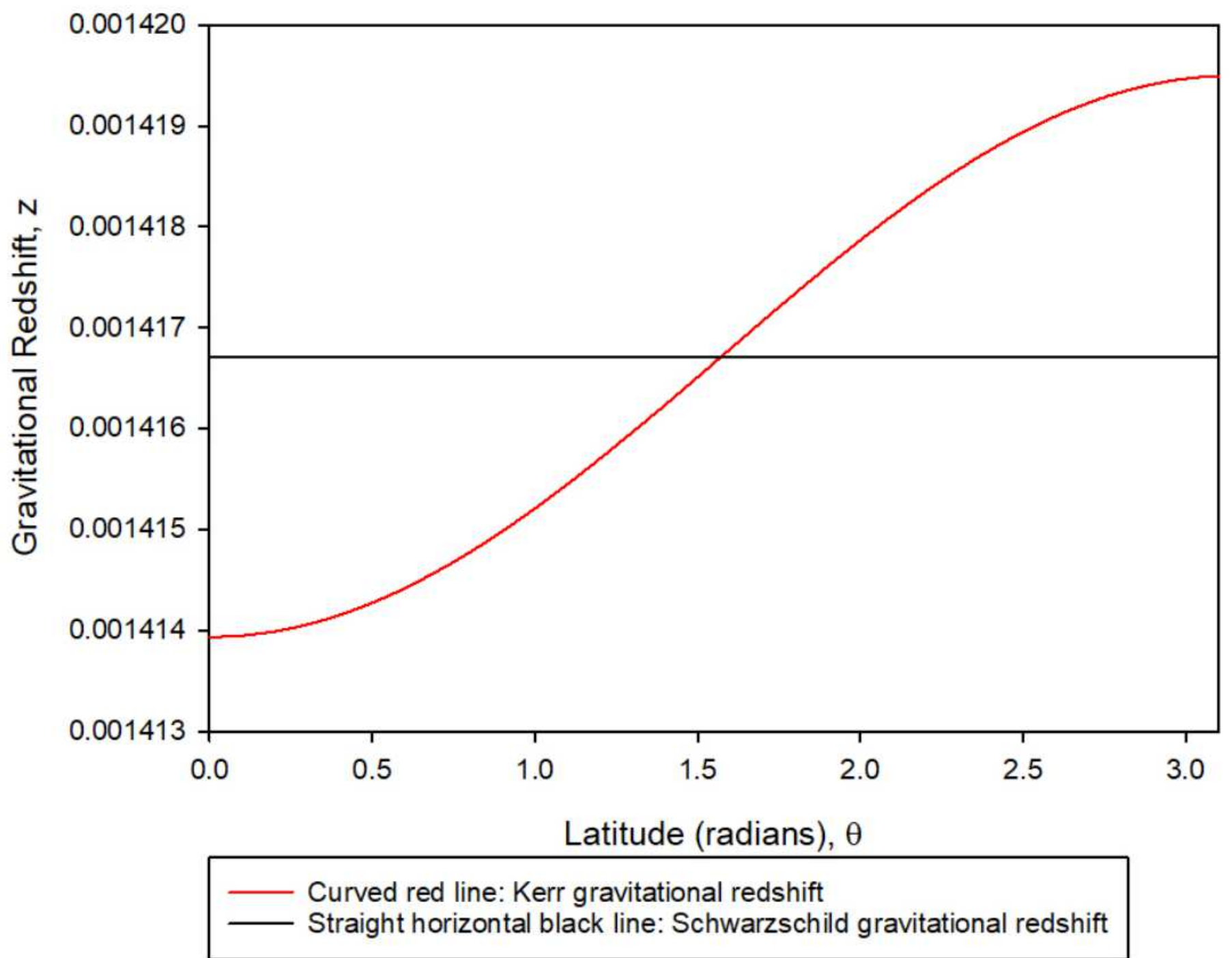

Figure 1

$\mathrm{z}$ vs $\theta$ for general relativistic white dwarf 
Fig, 2: z vs. $\theta$ for neutron star, XTE J1739-285

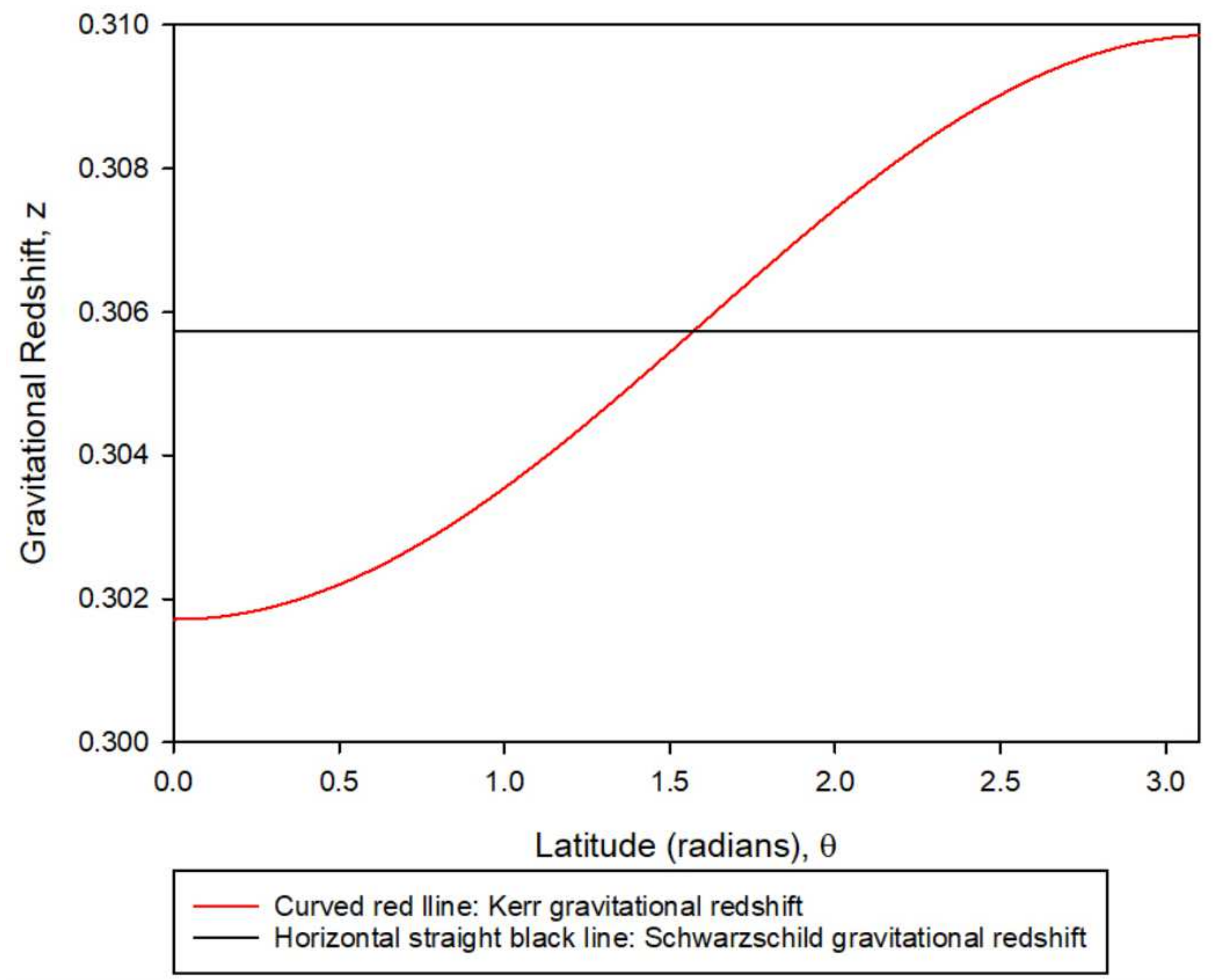

Figure 2

$z$ vs $\theta$ for neutron star, XTE J1739-285 